\title{
The initial segment strategy: A heuristic for route selection
}

\author{
JEREMY N. BAILENSON, MICHAEL S. SHUM, and DAVID H. UTTAL \\ Northwestern University, Evanston, Illinois
}

\begin{abstract}
People often choose one route when traveling from point A to point B and a different route when traveling from point B to point A. To explain these route asymmetries, we propose that people rely on a heuristic (the initial segment strategy, or ISS) during route planning. This heuristic involves basing decisions disproportionately on the straightness of the initial segments of the routes. Asymmetries arise because the characteristics that favor selection of a particular route in one direction will usually differ from those that favor selection when traveling in the opposite direction. Results from five experiments supported these claims. In the first three experiments, we found that subjects' decisions were asymmetric and involved a preference for initially straight routes. In Experiment 4, we confirmed that the ISS is a heuristic by demonstrating that people rely on it more when under time pressure. However, people can choose the optimal route when instructed to do so. In Experiment 5, we generalized the findings by having subjects select routes on maps of college campuses. Taken together, the results indicate that the ISS can account for asymmetries in route choices on both real and artificial maps.
\end{abstract}

A consistent finding in research on decision making and planning is that choices are often asymmetric. For example, people may judge that Cuba is more similar to the (former) Soviet Union than vice versa (Tversky, 1977). Asymmetries have been demonstrated in many different domains, including judgments of spatial distance (Holyoak \& Mah, 1982; McNamara, 1991; McNamara \& Diwadkar, 1997; Montello, 1991; Newcombe, Huttenlocher, Sandberg, \& Lie, 1996; Sadalla, Burroughs, \& Staplin, 1980), descriptions of networks (Denis \& Cocude, 1989; Robin \& Denis, 1991), and counterfactual reasoning (Dunning \& Parpal, 1989).

Our focus here is on asymmetries that occur when planning routes between two locations. Several researchers have demonstrated that route selections are often asymmetric; people consistently prefer a different route when traveling from location A to location B than when traveling in the opposite direction. These path asymmetries have been observed in many different kinds of tasks, including people's planning of routes from maps (Bailenson, Shum, \& Uttal, 1998; Christenfeld, 1995), drivers' recall of routes that they usually follow (Stern \& Leiser, 1988), and pedestrian's selection of routes when traveling across a parking

Portions of Experiment 5 were presented at the 20th annual meeting of the Cognitive Science Society and appear in its Proceedings. Portions of this work were supported by Grant R29 HD 34929 from the National Institute for Child Health and Human Development and LIS Grant 97201313 from the National Science Foundation. We thank John Coley and Juliabeth Proffitt for their helpful comments, Elissa Larkin, Alex Okrent, Layla Piland, and Stephanie Sun for running subjects, preparing stimuli, and entering data, Leah Vaughn for entering data, and Eleanor Antonucci and Wanda Shum for guidance. Correspondence concerning this article should be addressed to J. N. Bailenson, Department of Psychology, University of California, Santa Barbara, CA 93106-9660 (e-mail: bailenson@condor.psych.ucsb.edu). lot (Christenfeld, 1995) or along paths on a college campus (Shum, Bailenson, Hwang, Piland, \& Uttal, 1998).

Asymmetries in route selection are interesting because they involve a violation of what would seem to be a very important consideration in planning routes: the desire to take the shortest route. If people attempt to choose the shortest route and these attempts are successful, their selections should rarely be asymmetric; the shortest route will be the same, regardless of direction. However, people do not always choose the shortest route (Bailenson et al., 1998; Christenfeld, 1995). Rather, they seem to rely on heuristics for selecting routes. These heuristics minimize cognitive effort and usually will lead to satisfactory route choices. However, one consequence of the reliance on heuristics, as opposed to a systematic search for the optimal route, is predictable asymmetries. In the present work, we delineate the conditions under which people do and do not rely on a heuristic that leads to systematic asymmetries in route selections.

Christenfeld (1995) proposed one heuristic for route selection: People defer route decisions for as long as possible. As is shown in Figure 1, Christenfeld asked people to choose from routes that were identical in all aspects except the location along the route at which a turn was required. The layout of some possible routes required a turn early on, but on other routes, the turn could be deferred because of a long and straight initial segment. To minimize mental effort, people selected the routes on which the turn occurred last. Use of this heuristic leads to asymmetric decisions, because the route with the last turn will necessarily be different when traveling along a route in one direction than when traveling in the opposite direction. Christenfeld attributed his findings to the use of a domain-general decision-making heuristic for selecting among identical options. 


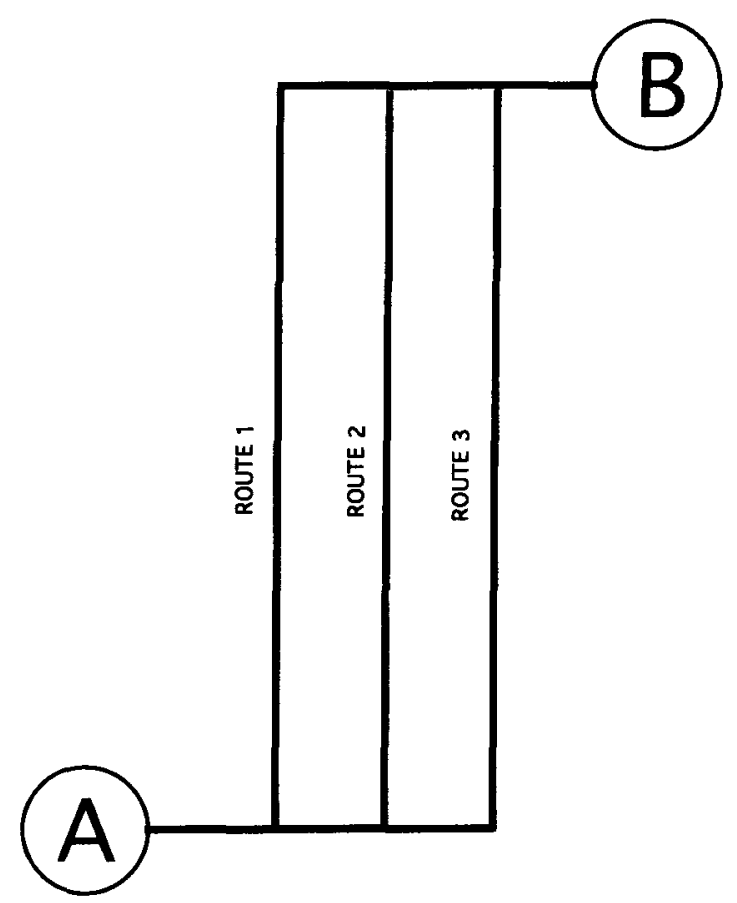

Figure 1. A depiction of the routes subjects traversed in Christenfeld's (1995) Experiment 5. When traveling from $A$ to $B$, subjects preferred Route 3 , because they did not have to turn until the last possible moment. When traveling from $B$ to $A$ they preferred Route 1.

Christenfeld's explanation adequately accounts for those situations in which the routes are identical except for the location at which a turn must be made. However, his model may not be applicable to many route choice situations. For example, two routes are rarely identical in all ways except the location of turns. Furthermore, people do not always have the option of delaying turn decisions. In many cases, the first route decision may be the only decision one has to make (i.e., deciding between two major highways for a long-distance trip). In these situations, there is no opportunity for people to delay decisions, yet asymmetries still occur (Shum et al., 1998). The purpose of the present paper was to provide a more general account of the factors that lead to asymmetries in route selection.

In this paper, as well as in previous work (Bailenson et al., 1998), we suggest that people tend to focus disproportionately on the initial portions of the routes (i.e., the segments near the origin). More specifically, people tend to make their route decisions on the basis of the straightness of the initial segments. As used here, straightness means the relative absence of curves or turns. We call this preference for initially straight route segments the initial segment strategy (ISS). Given the choice between two routes, people will prefer the one that is initially more straight, regardless of what the later portions of the routes look like. Use of this heuristic leads to path asymmetries, because a route may be initially straight at one end (near the origin), but not at the other end (near the destination). If origin and destination are switched (i.e., when someone makes a return trip), people may prefer a different route that is straight at their present location. This pattern of preferences will consistently lead to asymmetries.

Past research supports the notion that people attend particularly to the initial segments more than to the other segments. For example, Iida, Akiyama, and Uchida (1989; cited in Bovy \& Stern, 1990) asked people to assign travel time estimates to routes with which they were familiar. The characteristics of the initial portions of the routes had the largest effect on estimates of total travel times. For example, routes that began with sections that could be traveled quickly received consistently lower time estimates than routes on which the quickly traveled section came later. Furthermore, Bailenson et al. (1998) demonstrated that, when maps were segmented into distinct regions, people preferred routes that were initially straight over ones that contained a large number of turns in the initial portion, even when the initially straight routes were longer. People appeared to pay little attention to the appearance of the remainder of the routes.

In sum, the evidence from previous studies is consistent with the notion that asymmetries arise from a reliance on the ISS. The goals of the present work were (1) to provide a systematic explanation of why asymmetries in route choice occur, (2) to establish a coherent account of how use of the ISS may lead to route asymmetries, and (3) to thoroughly delineate the parameters of the ISS, including defining the meaning of key concepts, such as straightness and initial segment. We sought to answer questions such as the following. How straight does a segment need to be for subjects to choose asymmetrically? What proportion of the route is included in the initial segment? Is the initial segment defined by length along the route or by boundaries of a region? Do large route asymmetries occur on maps that are not bounded by regions?

In Experiment 1, we gave subjects an opportunity to choose between two routes of equal length, one that was relatively straight and one that contained various numbers of turns. We predicted that the subjects would prefer routes with fewer turns to routes that were less straight, even when the two routes were equal in length. In Experiment 2 , we demonstrated that people attend to the straightness of a route most often when the straight portion is at the beginning of the route. In this experiment, we manipulated the size and location of the straight portion and predicted that only the straightness of the initial portion (and not straight portions at other points in the route) would affect the subjects' preferences. In Experiment 3, we focused the subjects' attention on the initial portion of routes by imposing region boundaries on the maps. We predicted that we could shift the subjects' preferences for routes by changing the characteristics of route segments within the initial region. In other words, if the initial region were drawn so that the first turn in the route was included, the subjects should tend to avoid that route. If the initial region were drawn to exclude the first turn, then subjects should prefer that route. In this manner, varying the straightness of the initial portion of routes (without changing features of the actual routes themselves) 
should influence the decisions of subjects who are using the ISS.

In Experiment 4, we tested the claim that the ISS is a heuristic. Our claim is that the focus on initially straight segments is a strategy that people employ to reduce processing demands. Like other heuristics, the ISS does not reflect general constraints on the processing of information. Rather, it is a method that people rely on to reduce cognitive load (Payne, Bettman, \& Johnson, 1988; Simon, 1981). Therefore, in Experiment 4, we demonstrated (1) that people rely more on the ISS when under cognitive duress but (2) that they could select the optimal (i.e., the shortest) route when instructed to do so.

Finally, in Experiment 5, we attempted to generalize the findings by having subjects select routes on real maps of five college campuses.

\section{EXPERIMENT 1}

In Experiment 1, we tested the general idea that people prefer to travel on paths with fewer turns, regardless of the length of those routes. Although previous research has shown that travelers prefer routes containing fewer turns (Seneviante \& Morrall, 1986), to our knowledge, no studies have controlled for the absolute length of routes; usually, the more turns a route has, the longer it is. Because we claim that subjects' judgments are based specifically on the straightness of routes, it is necessary to provide direct evidence that people prefer straight routes even when the straight route is not shorter than the alternatives.

In the present experiment, the subjects chose routes on maps that contained two buildings and two possible paths between the buildings. One of the two paths on the map had more turns than the other path had, but the two paths were equal in length. Our account predicts that the subjects will systematically choose the route with fewer turns. A corollary prediction was that the magnitude of the preference for the route with fewer turns should be directly related to the number of turns in the alternate route: the more turns in the alternate, the greater the preference for the route with fewer turns.

\section{Method}

Subjects. The subjects were 20 Northwestern University undergraduates in an introductory psychology class, who participated to receive course credit.

Materials and Design. We created four map templates. As is shown in Figure 2, on each template, there was an origin building and a destination building, joined by two possible routes. The routes did not intersect; consequently, once a person selected a particular route, he or she had to follow that route until the destination was reached. On two of the templates, the paths spanned from top-left to bottom-right, whereas on the other two templates, the paths spanned from top-right to bottom-left. One of the routes that joined the two buildings featured a single turn (single-turn route). The other route (multiple-turn route) was always the same length as the single-turn route but had more turns. For each template, we created four versions, in which we varied the number of turns in the multipleturn route-two, three, four, or five turns. As a result, there were four versions of each template (resulting in 16 different maps).
Each subject received a page of instructions and a booklet consisting of 32 pages. The first 16 pages of the booklet contained all four map templates in all of the number-of-turns conditions in a random order. The final 16 pages were simply copies of pages 1 to 16. Thus, each subject saw each map twice. Half of the booklets depicted the origin building on top, whereas the other half depicted the destination building on top. We manipulated the main variable of interest, the number of turns in the multiple-turn route, within subjects. Every subject saw the single-turn route pitted against a multipleturn route that contained two, three, four, and five turns.

Procedure. The subjects participated in groups of 12 or less. The instructions were as follows:

In the following experiment, you will see a series of maps of a town.
Each map is on a separate page. Thick dark lines on the map represent
roads on which you can travel; you will also see buildings and lakes on
the maps. Your task for each map is to find a route between two build-
ings. The letter "S" appears on the building you must start from. Your
destination is the building which is denoted with the letter "F." So your
job is to find a path from "S" to "F." While you travel, you must stay on
the streets (thick dark lines). Please begin on a street which emerges
from the building indicated by "S." Please do not look back over maps
that you have completed already.

The subjects indicated their choice by tracing the route with a highlighter. After they completed the packet of maps, the subjects completed a postexperiment questionnaire that asked their age, gender, and handedness.

\section{Results and Discussion}

The dependent variable was the percentage of times the subjects selected the single-turn route over the multipleturn route. Over all trials, the subjects selected the singleturn route $80 \%$ of the time. This percentage differed significantly from what would be expected from chance $(50 \%)$ $[t(19)=6.48, p<.05]$. We were interested in whether this percentage varied depending on the number of turns in the multiple-turn route. There was a significant linear trend $[F(1,19)=5.35, p<.01]$. As the number of turns in the multiple-turn route increased, the subjects' preference for the single-turn route increased. The means for maps with two, three, four, and five turns were $.21, .24, .20$, and .13, respectively. The subjects performed comparably on the first and the second presentations of the maps. ${ }^{1}$

The results of Experiment 1 are consistent with Seneviante and Morrall's (1986) finding that subjects prefer straight paths over alternatives that contain turns. Moreover, the present experiment shows that this advantage holds even when the length of the two paths is held constant. The results thus replicate prior findings that have shown that people overwhelmingly prefer straight routes. Moreover, the present findings indicate that straightness can be more important than length in determining which route people will select. In Experiment 2, we went on to test whether people base their decisions on straightness more often when the straight segment of the route occurs near the origin than when the straight segment occurs in other places along the route.

\section{EXPERIMENT 2}

A crucial element of the ISS is that straight segments should have more influence when they occur near the ori- 


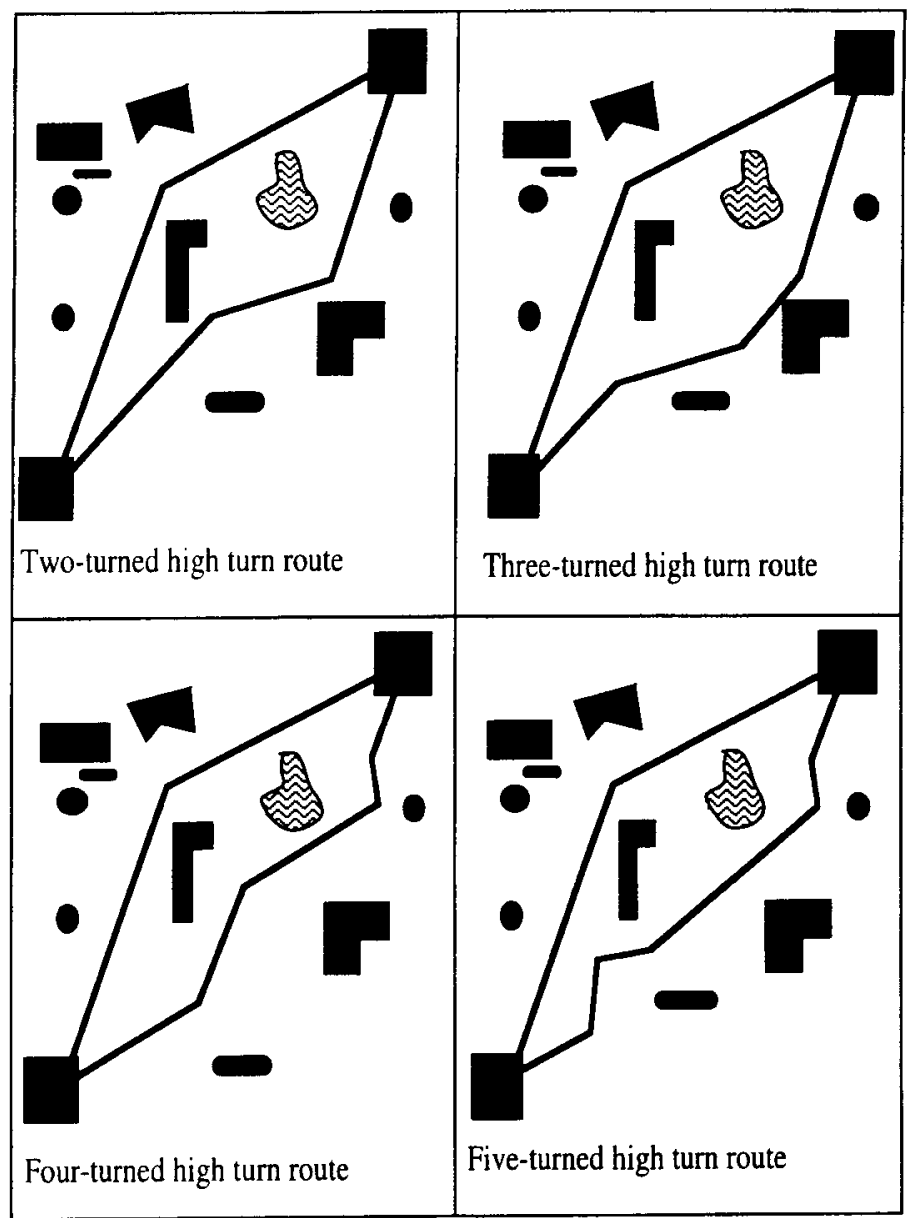

Figure 2. Four different versions of one of the maps used in Experiment 1. There were two possible routes-a single-turn route that contained only one turn, and a multiple-turn route that contained more than one turn. As we increased the number of turns in the multiple-turn route, the subjects were more likely to select the single-turn alternative.

gins than when they occur in other places along the route. Recall that we claim that asymmetries arise in route selections from a preference for routes that begin with straight segments. In Experiment 2, we tested this claim. We also attempted to specify more clearly what constitutes an initial segment. We asked people to choose between two routes that were identical in all aspects (including length and overall number of turns) except the straightness of the initial segment. One end of the two paths was straight, whereas the other end contained a number of turns and curves. We predicted that the subjects would prefer the route that began with the initially straight segment and that their choices, therefore, would be asymmetric.

In addition, we investigated whether portions of routes other than the initial segment affected the subjects' route preferences. To test for this, we varied how much of the routes was straight and how much of the routes contained turns. If people focus most on the initial segments of the route, characteristics beyond the initial segment should have relatively little effect. What should matter most is the straightness of the initial portion of the route, not the overall straightness of the route.

\section{Method}

Subjects. The subjects were 23 Northwestern University undergraduates in an introductory psychology class, who participated to earn partial course credit.

Materials and Design. We created two map templates. On each template, there was an origin building and a destination building that were joined by two routes. The two paths were actually identical; one was simply rotated $180^{\circ}$, relative to the other. Consequently, on each map, there were two routes. The initially straight (IS) route began with a straight section and ended with a curved section. The initially circuitous (IC) route began with a curved section and ended with a straight section. As in Experiment 1, the paths did not intersect.

From each of the two templates, we created four maps (see Figure 3). Across the four maps, we varied how much of each route was straight and how much of each route contained turns. On the control map, both routes contained turns for the entire route. For the three experimental maps, all of the routes contained a straight 


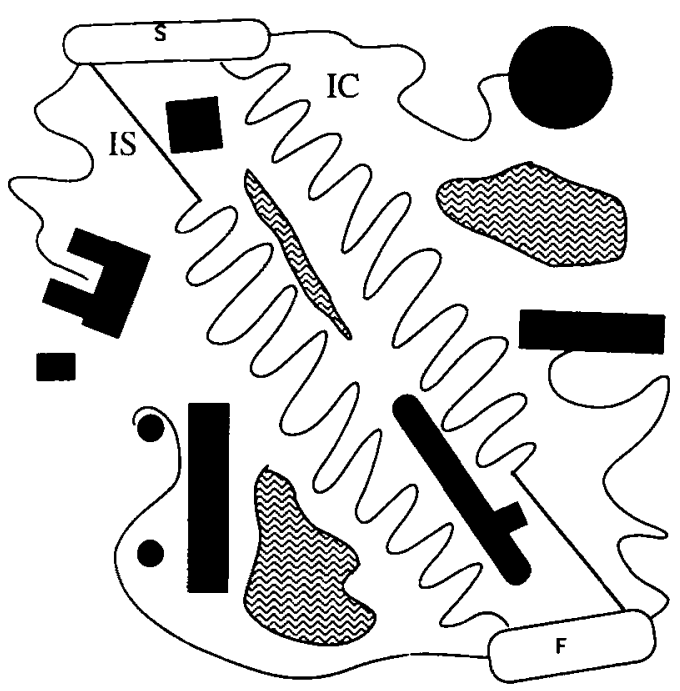

Figure 3. One of the four experimental maps used in Experiment 2. This particular map consists of routes that are $25 \%$ straight. The initially straight route is labeled "IS," and the initially circuitous route is labeled "IC."

segment at either the end or the beginning of the route. The straight segment was always at least one quarter of the routes. We varied the percentage of the remainder of the routes that contained turns: about $50 \%$, about $67 \%$, or almost $100 \%$. For each map template, the number of actual turns and the width of the turns were held constant across conditions.

The test booklets were organized as follows. After the initial instruction page, there were 16 maps, each appearing on a separate page. The maps were organized into two blocks of 8 . In the first block, a subject saw all 8 maps (the four versions of each of the two templates) in a random order. All of the maps in the first block had either the building marked " $\mathrm{S}$ " (the origin) near the top of the map or the building marked " $F$ " (the destination) near the top of the map (see Figure 3 ). In the second block, the subjects saw the 8 maps in the same order as that in Block 1; however, the origin and the destination were switched (i.e., if the origin had been on top in the first block, the destination was on top in the second block). The locations of the origin and the destination buildings were counterbalanced. Half of the subjects received maps with the origin near the top in the first block, and the other half received maps with the destination near the top in the first block.

An important characteristic of the design was that it allowed an assessment of both within- and between-subjects asymmetries in route selection. Detection of within-subjects asymmetries involved examining both blocks to determine when the subjects' selection of an alternate route had depended entirely on whether the straight segment of the route was near the origin or near the destination. Detection of between-subjects asymmetries involved examining whether there was a consistent preference across subjects for routes that had straight sections near the origin. For this analysis, we examined only the first block, because selections made in the second block conceivably could be influenced by what people chose in the first block.

Procedure. The instructions and procedure were identical to those of Experiment 1.

\section{Results and Discussion}

The subjects showed no preference for the left route (chosen on $47 \%$ of the trials) or the right route (chosen on $53 \%$ of the trials). However, for the experimental maps, the subjects consistently preferred the routes that began with initially straight sections. As had been predicted, these preferences led to asymmetries in route selections.

The main dependent variable for the experimental maps was the percentage of trials on which subjects preferred the IS route, as opposed to the IC route. Over all trials in the three experimental conditions, the subjects preferred the IS route $64 \%$ of the time. This percentage differed significantly from chance $[50 \% ; t(22)=2.31, p<.05]$. This result reveals within-subjects asymmetries. For any given subject, each route was an IS route half of the time and an IC route the other half of the time, depending on the placement of the origin and the destination. Thus, the subjects switched their selection of routes, depending on which point was the origin and which the destination, even though the two routes were identical. That is, on the same map, the same subjects tended to choose different routes, depending on the placement of the origin and the destination.

To examine the presence of between-subjects asymmetries, we looked at the subjects' performance on just the first block of experimental trials. On these trials, the subjects selected the IS route $75 \%$ of the time. Thus, the subjects consistently preferred an IS route, regardless of whether the origin was near the top or the bottom of the map.

These results suggest that what matters to subjects in route selection is not the overall curviness of the route but, rather, the straightness of the initial portion of the route: If the initial portion is straight, subjects will prefer it, regardless of how circuitous the rest of the route is. Recall that we included three experimental conditions that varied the overall curviness of the portions of the routes adjacent to the straight segment. However, this manipulation did not significantly affect the subjects' preferences: The percentage of trials on which the subjects selected the IS route was $75 \%, 74 \%$, and $76 \%$ for the maps that contained $50 \%$ turns, $67 \%$ turns, and $100 \%$ turns on the remainder of the routes, respectively.

These results provide a particularly compelling demonstration of the effects of the initial portions of routes on subjects' preferences. The consistent preference for the routes that began with a straight section, regardless of what happened in the remainder of the route, contrasts with the control condition routes, in which subjects demonstrated no preference for the left or the right routes. That subjects switched their path choice solely because of the features of the initial segment provides strong support for our account of asymmetries and route choice. The initial straightness of routes appears to be crucial in determining which route a person will select.

\section{EXPERIMENT 3}

The results of the prior experiments demonstrate (1) that the straightness of routes influences people's preference and (2) that people prefer routes with IS segments to routes where the straight segments occur in other places along the route. In Experiment 3, we sought to provide additional evidence for the disproportionate influence of 
the initial portion of routes. We attempted to manipulate the initial portions of routes by inserting regions and boundaries onto the map. Previous work (e.g., Bailenson et al., 1998; Downs, Liben, \& Daggs, 1988; Sadalla et al., 1980) has shown that people tend to make route decisions on a region-by-region basis when a map is segmented into a number of bordering areas. That is, people focus on route choices within a given region and follow the selected route until they reach the next region, where they repeat the decision-making process.

We hypothesized that adding regions would change people's perceptions of what constituted an initially straight route segment. Specifically, we predicted that people would prefer initial route segments that did not contain a turn within the initial region, even when the overall straightness and length of the alternatives were held constant. The origin regions were designed to include a turn on one route and none on the other, or vice versa. We predicted that the subjects would prefer paths that did not contain turns in the initial regions over those that did contain turns, although the paths themselves were identical. In addition, we predicted that this preference would shift when we reconfigured the regional boundaries to either include or exclude a turn in the initial route segments. In sum, we predicted that we could alter people's route selections simply by changing whether the initial region contained a turn. This finding would provide strong support for the claim that people focus differentially on the straightness of initial segments and that these preferences contribute to the occurrence of asymmetries.

\section{Method}

Subjects. The subjects were 22 undergraduates in an introductory psychology class, who participated to earn partial course credit.

Materials. We created four map templates. On each template, there was an origin building and a destination building that were joined by two possible routes. The two routes on each map were identical, except that one was flipped $180^{\circ}$, relative to the other (see Figure 4).

Each template was varied in two ways. First, we varied whether the origin or the destination appeared on top. Second, we varied the configurations of the regions on the map; half of the paths on the maps had turns in the initial region, and the other half did not. More specifically, on half of the maps, the path on the left contained turns in the initial region, whereas the path on the right contained no turns in the initial region. The location (left or right side of the page) of the paths that did and did not contain turns in the initial region was reversed for the remainder of the maps. Consequently, there were four versions of each of the four templates, resulting in 16 different maps. An example of the two different regionalization schemes is shown in Figure 4.

Counterbalancing. A packet consisted of an instruction page and 16 maps. The maps were organized into blocks so that each subject saw four separate blocks. The order of blocks was constrained so that the first two blocks always featured either the origin on top for both blocks or the destination on top for both blocks. The third and fourth blocks in the packet always featured the opposite: If the first two blocks in the packet featured the origin on top, the second two blocks in the packet featured the destination on top. Order of the blocks was counterbalanced so that half of the subjects received the origin on top first, whereas the other half received the destination on top first.
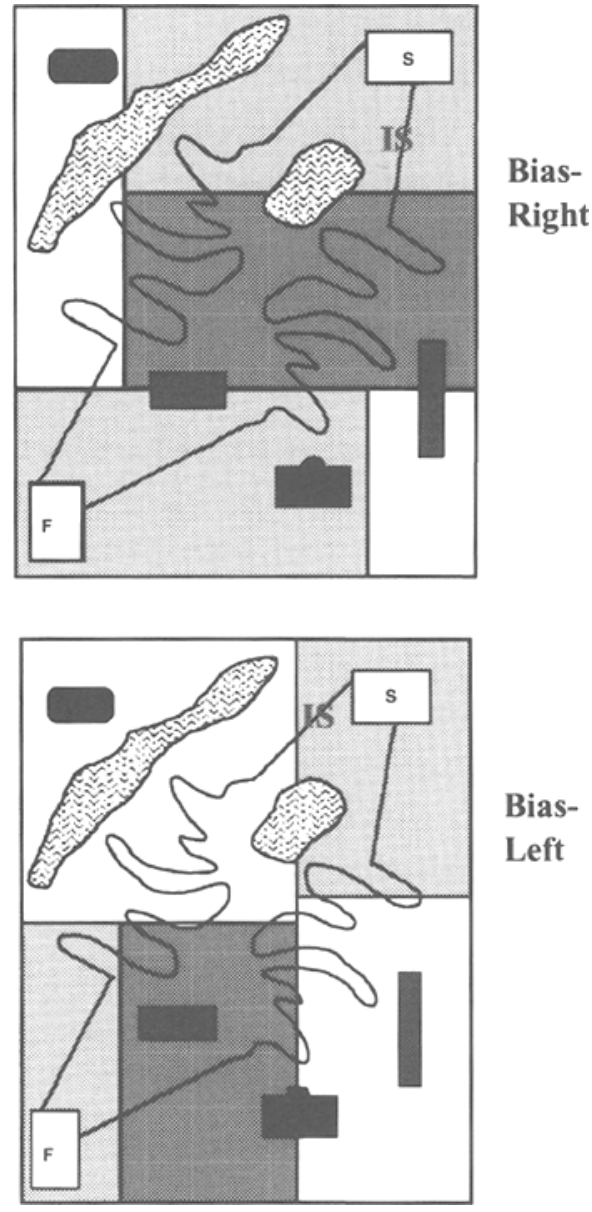

Bias-

Left

Figure 4. The two regionalized versions of one of the four experimental maps used in Experiment 3. The maps were identical, except for the configuration of the regions. The shape of the first region determined which of the two routes the subjects were likely to select. The top map is labeled "Bias-Right," because the route on the right contains no turns in the initial region. The bottom map is labeled "Bias-Left," because the route on the left contains no turns in the initial region. When the origin and destination labels ("S" and " $F$ ") were reversed, the bias was switched from one route to the other.

Procedure. The procedure was similar to that used in the previous experiments.

\section{Results and Discussion}

We predicted an overall preference for routes that contained no turns in the origin region. Consequently, the dependent variable was the percentage of times the subjects selected the route that did not contain a turn in the initial region. Over all trials, the subjects selected the predicted route $58 \%$ of the time. This differs significantly from what would be expected by chance $[t(19)=2.35, p<.05]$. In addition, the subjects selected the predicted route over $60 \%$ of the time on three out of the four map templates. This finding indicates that the particular configuration of the origin region on a map affected what routes people preferred. Recall that, on each map, the only difference between the two possible routes was whether the initial re- 
gion contained a turn. The subjects reliably preferred the routes that did not contain turns in the initial segment.

Imposing regions on maps focused the subjects' attention, to some degree, on the initial region containing the origin. Selection was based on the straightness of the available routes in that initial region. As was shown in Experiment 1, subjects preferred straight routes over ones containing turns. Experiment 2 demonstrated that this preference is based on the initial straightness of the route. The present experiment confirmed both of these findings. Even when the routes were identical in shape and length, focusing a person's attention on the straight portion of the initial segment determined which route he or she chose.

In Experiment 4, we sought to provide evidence that the ISS can be construed as a heuristic. We predicted that use of the ISS would increase when time constraints were imposed but would decrease when subjects were encouraged to choose the shortest route.

\section{EXPERIMENT 4}

We claim that the ISS is a heuristic-a rule-of-thumb that leads to suboptimal but generally satisfactory route selections. This claim suggests that people do have the ability to pick the optimal (i.e., the shortest) route; however, they attempt to minimize mental effort by attending disproportionately to the initial segment. Previous research has suggested that heuristics are used in situations in which mental resources are taxed, such as when there is not time to work out the optimal solution (Payne et al., 1988; Simon, 1981). To provide support for this notion, we flashed the maps quickly on a computer screen and then asked the subjects to choose a route. We predicted that, under this limited processing time, the subjects would resort to the ISS more often than when they had unlimited time.

Furthermore, we also tested whether the subjects were capable of selecting the shortest route. We predicted that, when given instructions to do so, the subjects could select the optimal route, even under time constraints. This pattern of results would confirm that people rely on the ISS to minimize cognitive effort but that they are, in fact, capable of putting it aside when they want to make an optimal decision.

\section{Method}

Subjects. The subjects were 60 Northwestern University undergraduates in an introductory psychology class, who participated to earn partial course credit.

Materials. We created 4 experimental maps and 24 filler maps. Each map was designed to completely fill the screen of a 14-in. computer monitor. The maps were designed to control for various factors, such as the relative positions of routes on the computer screen. On each map, one building was marked with START (the origin), and one was marked with FINISH (the destination).

Each map contained three nonintersecting routes from the origin to the destination (see Figure 5). As in Experiment 2, the experimental maps were designed to test the hypothesis that people would prefer paths that began with initially long, straight sections, even if these paths were longer than the alternatives. We varied the characteristics of the three routes as follows. Two of the three routes tested our ISS hypothesis. The IS route always began with a long, straight initial segment and ended with a circuitous portion. The IC route began with a curved section and ended with the straight section. As in Experiment 2 , we simply flipped the initially straight route on the horizontal axis. Therefore, the IS route when traveling in one direction was actually the IC route when traveling in the opposite direction. We also included a third route, the shortest ( $\mathrm{SHO}$ ) route, on each map, which was the shortest (Euclidean) distance from the origin to the destination. The SHO route did not contain any straight segments that were greater than $15 \%$ of the length of the other routes. The SHO route was constructed by connecting the origin and the destination with a line that was approximately one third shorter than the other two routes. Consequently, we predicted that the subjects would not choose the SHO route if they relied on the ISS. Two of the four experimental maps featured the SHO route in the center of the screen (i.e., between the IS and the IC routes), and the remaining two experimental maps featured the SHO route on one of the sides of the screen.

The filler maps were used to prevent the subjects from discerning the nature of the study. They were similar to the experimental maps in that they had an origin and a destination that were connected by three nonintersecting routes. On the filler maps, the characteristics of the three routes, such as the length of the routes and the number of turns, were varied randomly.

Design. We varied two factors between subjects. The first factor was the amount of time the subjects were allowed to look at each map. Two of the groups viewed maps for a limited amount of time before making their decisions (the timed condition), and the other two groups had as much time as they wanted to examine the maps (the untimed condition).

The second factor was whether the subjects were given a specific goal; that is, whether they were explicitly instructed to choose the optimal "shortest" route. This factor was included to demonstrate that subjects could pick the optimal route even in the timed conditions. This finding would provide further evidence that the ISS was a heuristic that people relied on when they were not motivated to determine an optimal solution. Half of the subjects in the timed condition and half of the subjects in the untimed condition were assigned to the goal condition; the remaining subjects were assigned to the nongoal condition. The crossing of the two factors resulted in a total of four conditions.

In all of the conditions, the subjects saw each experimental map twice, once with START near the top of the screen and once with START near the bottom. Thus, there were 8 experimental trials (two directions on each of 4 maps). In addition there were 24 filler maps, resulting in 32 trials total. Each subject saw the 32 trials in a different random order.

Procedure. All the subjects were told that they would be asked to find a route between a "start location" and a "finish location." They were told that, on each map, there would be three distinct routes from which they could choose: the one on the right, the one in the middle, and the one on the left. The subjects were instructed to press separate keys corresponding to each of these three choices.

Each of the 32 trials began with a ready prompt, which signaled subjects that a new trial was about to commence. This prompt stayed on the screen until the subjects pressed a key to continue with the trial. The second screen was the location prompt, which indicated to subjects the approximate location of the origin building (either START ON TOP Or START ON BOTTOM). The location of this prompt on the screen depended on the location of the origin building on the subsequent map (i.e., START ON TOP appeared near the top of the screen, and START ON BOTTOM appeared near the bottom of the screen). The location prompt appeared on the screen for $2,000 \mathrm{msec}$ and disappeared. The map was then displayed on the screen (for $750 \mathrm{msec}$ in the timed condition, and until subjects hit the spacebar in the untimed condition). After the map disappeared, the subjects were asked to respond by hitting a key corresponding to the route they selected to go from START to FINISH (" $z$ " for the leftmost route, " $x$ " for the center route, and " $c$ " for the rightmost route). 

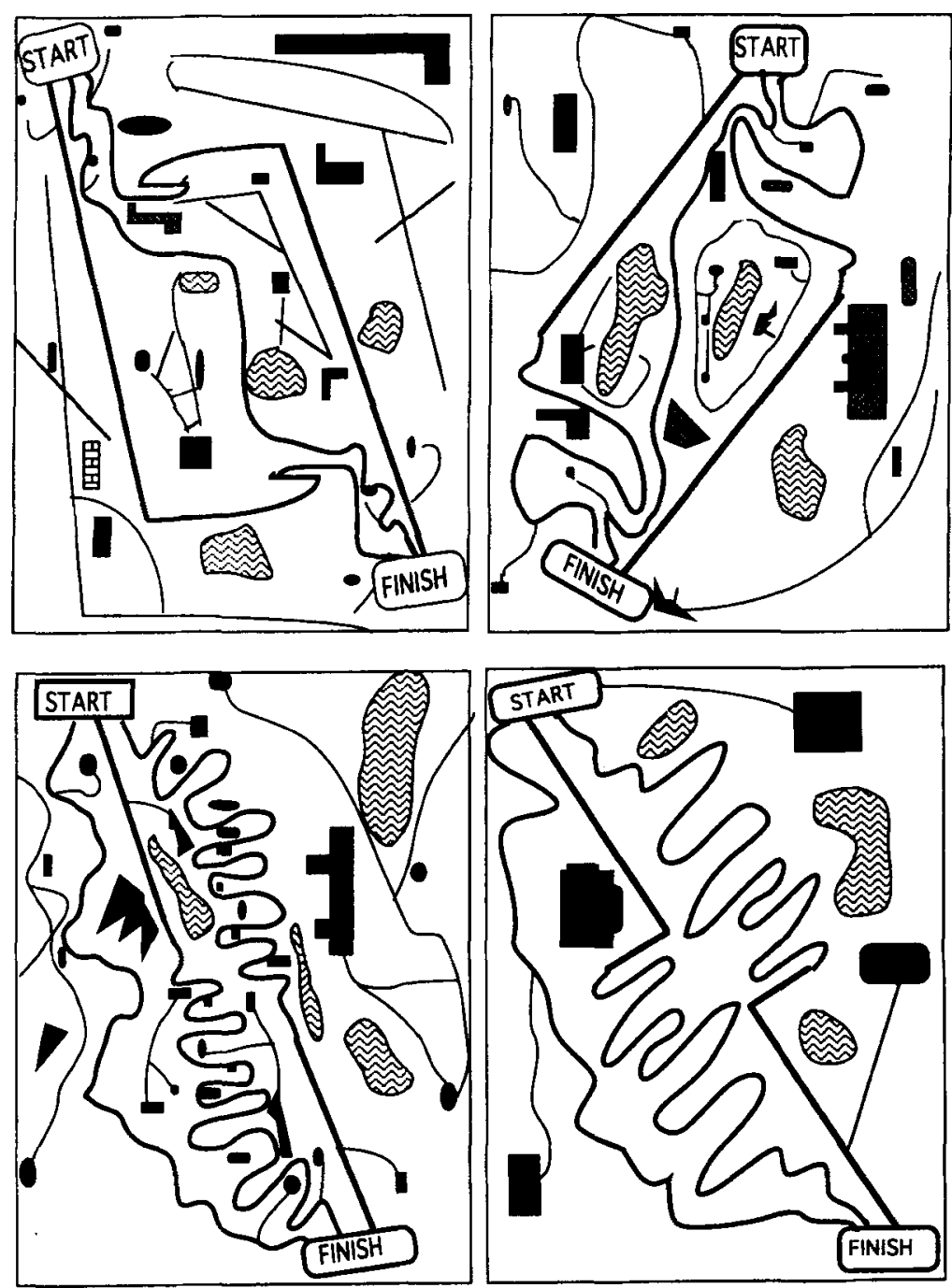

Figure 5. The four experimental maps used in Experiment 4. There were three possible routes on each map: the optimal (shortest) route, the initially straight route (IS), and the initially circuitous (IC) route. Note that the latter two routes are identical, except that they were flipped on the horizontal axis. Which of the two was the IS route (and which the IC route) was determined by the placement of the origin and destination.

To help familiarize the subjects with the procedures, there were 4 practice trials. Prior to the practice trials, the subjects in the goal condition were told: "One of three routes on each map is shorter than the other two. Your goal in this study is to attempt to choose the shortest route on each map." The maps in the 4 practice trials were similar to those used as filler maps in the actual experiment. At the end of the 32 trials, the subjects were asked to answer a short questionnaire concerning their age, gender, handedness, and whether they had seen the same map more than once.

\section{Results}

Table 1 shows the percentage of times the subjects selected the IS, IC, and SHO routes in each of the four conditions (timed-nongoal, untimed-nongoal, timed-goal, and untimed-goal). To ascertain whether there were any differences in performance between the four groups, we conducted a 2 (time) $\times 2$ (goal) $\times 2$ (route choice) analysis of variance (ANOVA), with percentage of IS and SHO responses as the dependent variables. Time (time vs. untimed) and goal (goal vs. nongoal) were betweensubjects factors; route choice was a within-subjects variable that represented the difference between the percentages of IS and SHO responses. We excluded IC responses from the ANOVA, because we were primarily interested in the difference in response between a route consistent with the ISS (the IS route) versus the SHO route. The results were as follows.

Time. If the ISS is indeed a heuristic, subjects should rely on it more when they are under a time constraint. Thus, we predicted that the subjects would select the IS route more often in the timed than in the untimed condi- 
Table 1

Percentage of Trials on Which Subjects Selected the Initially Straight (IS), Initially Circuitous (IC), and Shortest (SHO) Routes in Each of the Four Conditions in Experiment 4

\begin{tabular}{lrcc}
\hline & \multicolumn{3}{c}{ Route Selected } \\
\cline { 2 - 4 } Condition & IS & IC & SHO \\
\hline Timed-nongoal & 37 & 22 & 41 \\
Untimed-nongoal & 8 & 10 & 83 \\
Timed-goal & 17 & 17 & 66 \\
Untimed-goal & 11 & 1 & 88 \\
All conditions & 18 & 13 & 70 \\
\hline
\end{tabular}

tion. This prediction was confirmed by a significant interaction between route choice (the difference between IS and SHO responses) and time (timed vs. untimed; $F(1,56)=30.30, p<.05]$. The subjects selected the IS route more often in the timed conditions than in the untimed conditions ( $27 \%$ vs. $9 \%$, respectively). They also selected the SHO route less often in the timed than in the untimed conditions ( $53 \%$ vs. $85 \%$, respectively).

Goal. In addition, we predicted that subjects are capable of selecting the SHO route, even under time constraints, when they are explicitly asked to do so. The results confirmed the prediction that people could choose the $\mathrm{SHO}$ route; there was a significant interaction between route choice and goal [goal vs. nongoal; $F(1,56)=7.01$, $p<.05]$. The subjects selected the IS route more often in the nongoal conditions than in the goal conditions $(22 \%$ vs. $14 \%$, respectively). They selected the SHO route less often in the nongoal than in the goal conditions ( $62 \%$ vs. $77 \%$, respectively). In sum, when the subjects were explicitly asked to find the SHO route, they were able to do so, even under the timed condition in which maps were presented for $750 \mathrm{msec}$. This suggests that the ISS is indeed a heuristic that subjects can selectively employ.

Time $\times$ goal. Finally, we found a significant interaction between the two between-subjects factors (time and goal) and route choice $[F(1,56)=5.50, p<.05]$. As is shown in Table 1, the magnitude of the increase in use of the ISS when under a time constraint depended on whether the subjects were attempting to find the shortest route. The subjects were more likely to select the IS route in the timed than in the untimed conditions. However, this difference was much greater when the subjects were given a goal $(29 \%$ difference between the timed and the untimed conditions) than when they were not given a goal ( $6 \%$ difference). In other words, the subjects relied on the ISS most when they were placed under a time constraint and when they were not explicitly asked to find the shortest route.

Given that subjects can select the optimal route even when presented with maps for only $750 \mathrm{msec}$ (66\% optimal choices in the timed-goal condition), our results are quite striking. The subjects selected the IS route virtually as often as the optimal route ( $37 \%$ vs. $41 \%$ ) when not given a goal in the timed condition. In this experiment, we controlled the length of the routes so that the IS and IC routes were $50 \%$ longer than the SHO routes, yet we still found asymmetries owing to subjects forgoing the optimal route.
The results of the previous four experiments raise the question of whether they would generalize to maps that people actually use in the real world. We therefore decided to conduct an additional experiment with less stringently controlled but more externally valid stimuli. In Experiment 5, we asked subjects to plan routes on real maps of college campuses.

\section{EXPERIMENT 5}

The results of the first four experiments have shown that people rely on the ISS when choosing routes on maps. However, these maps were constructed specifically for use in our experiments. This raises the question of whether asymmetries that stem from the reliance on the ISS also occur when people choose routes on actual maps. This is an important question, because on most of the maps used in the previous studies, the subjects faced only one or two alternatives. In real route-planning situations, people may be forced to choose among multiple alternatives.

Therefore, we sought, in Experiment 5, to determine whether the prior results would generalize to actual maps that people use in everyday route-planning tasks. We selected campus maps from American universities on the basis of several criteria (discussed below). We chose travel situations in which there were at least two distinct routes joining two buildings or areas and then asked the subjects to choose routes between the two points. We predicted that there would be path asymmetries in planning routes and that these asymmetries would result from the use of the ISS heuristic.

\section{Method}

Subjects. The subjects were 32 Northwestern University undergraduates in an introductory psychology class, who participated to earn partial experimental credit.

Materials. We chose maps of campuses that met the following criteria: First, there were two clear buildings that were joined by two or more distinct paths. Second, there was limited access to the paths; once a traveler chose a path, there were not many opportunities to exit that specific path. Third, each of the two connecting routes conformed to our depiction of an ISS route relative to one of the buildings. In other words, one of the routes had a long straight segment near one of the buildings, whereas the other route had a long straight segment near the other building.

We looked at 25 campus maps in total. Each map was rated independently by the experimenters on each of the three criteria. The five maps that were rated highest in terms of meeting the criteria were selected to be the experimental maps. The five maps that were rated lowest in terms of meeting the criteria were selected to be the filler maps.

Figure 6 shows one of the experimental maps (University of Massachusetts at Amherst). The routes in question concern the loop existing between two buildings, one at the intersection of Commonwealth Ave. and Massachusetts Ave. (point A) and one at the intersection of Governor's Drive and N. Pleasant St. (point B). The subjects in the experiment were asked to find a route between points $A$ and $B$, half the time with $A$ as the origin and half the time with $B$ as the origin. Notice, in Figure 6, two potential routes between A and $B$, the first involving Commonwealth Ave. and Governor's Drive (Route $1,20.96 \mathrm{~cm}$ in length), and the other involving N. Pleasant St. and Massachusetts Ave. (Route 2, $22.71 \mathrm{~cm}$ ). The route that we predicted the subjects would select depends on whether A or B is the origin. 
Design. Each subject saw a packet of 20 maps, organized into two blocks. In the first block, the subjects saw all 5 experimental maps, along with the 5 filler maps. For each map, we denoted the origin with a sticker showing the letter "S," while we denoted the destination with the letter "F." The order of the fillers and the ex- perimental maps was randomized. In the second block, the subjects saw the same 5 experimental maps; however, the origin and the destination buildings were reversed. We designed the filler maps to disguise the goal of the experiment. On these maps, the origins and destinations were denoted with entirely separate buildings than

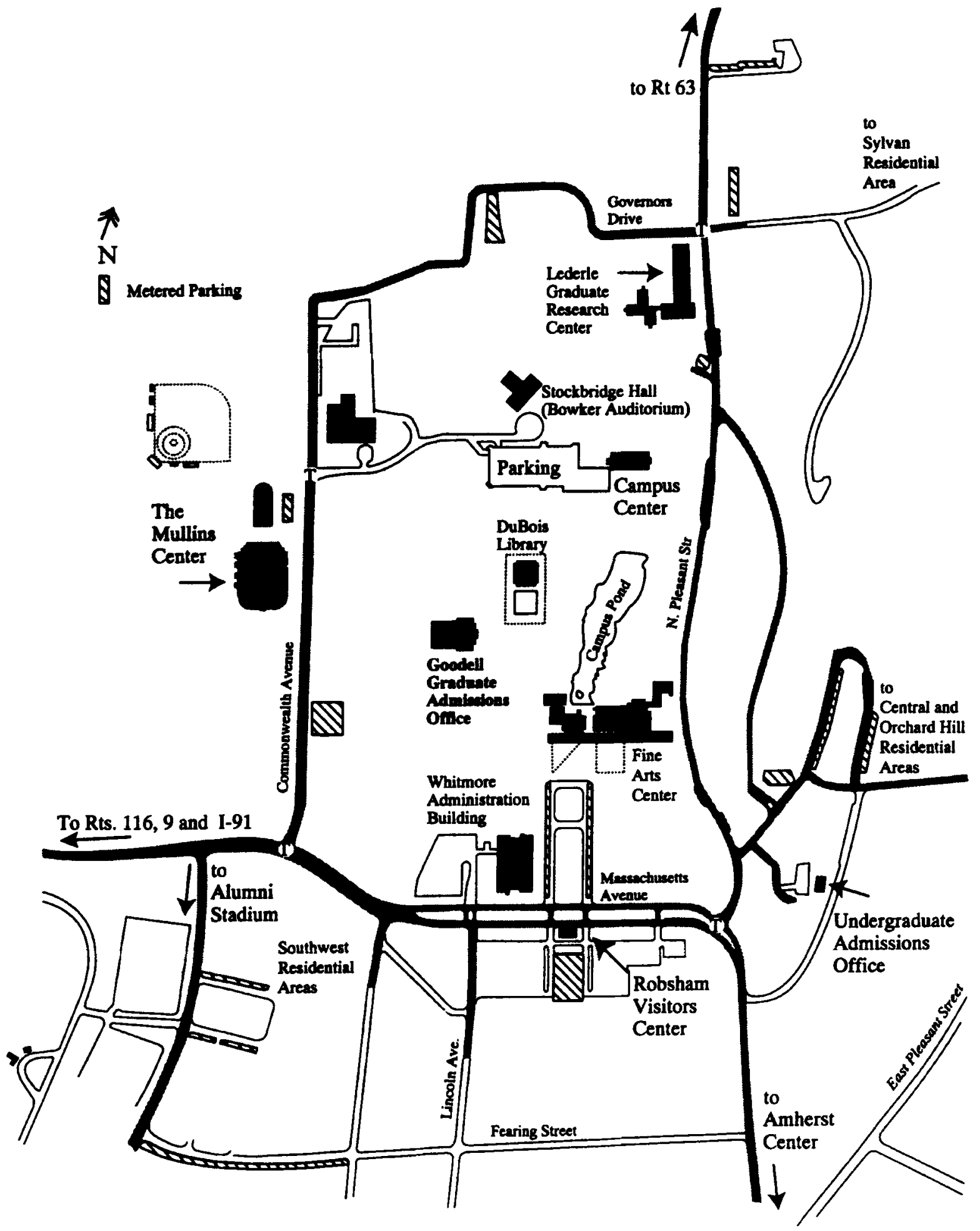

Figure 6. The actual Amherst map used in Experiment 5. The top of the page is north. 
those in the first block. The ordering of the maps was the same in both blocks. The procedure was identical to that used in the first three experiments.

\section{Results}

The dependent variable was the percentage of times the subjects selected the predicted ISS route, as opposed to any other route available. Over all experimental trials, the subjects selected the ISS route $56 \%$ of the time. We wanted to compare this level of responding with what would be expected by chance alone. We therefore estimated the percentage of chance responding by asking a separate group of 10 subjects to count the number of "reasonable routes that a traveler might take" between Points A and B on each of the five experimental maps. Chance was calculated by dividing $100 \%$ by the mean number of routes proposed for each map. For example, if there were five possible routes between Points $\mathrm{A}$ and $\mathrm{B}$, chance responding for each route on that map would be $20 \%$. The mean level of chance responding for the five experimental maps was $30 \%$. The $56 \%$ level of response for ISS-consistent routes was greater than this $30 \%$ level of chance when choices were computed across subjects $[t(31)=13.23]$ and for individual map items $[t(4)=2.95$, both $p s<.05]$.

Table 2 shows the route choices made by the subjects on each of the five experimental maps and also the chance level of responding for each map. The percentages indicate the proportion of subjects who selected the ISS route in that particular condition. Remember that, on each map, the subjects were asked to find two routes: once going from Point A to Point B, and once going from Point B to Point A. In Table 2, we arbitrarily classified the routes chosen on these two routes by the general direction of the route: either traveling north or east $(\mathrm{N} / \mathrm{E})$ or south or west $(\mathrm{S} / \mathrm{W})$. Note that, on each of the five experimental maps, the subjects selected the ISS-consistent route overall more often than they would by chance alone.

Examining the results for the experimental maps individually highlights the effects of straight initial segments on route preferences. Consider, for example, the routes on the Amherst map shown in Figure 6. When heading from Point A to B, Route 1 (Commonwealth Ave. to Governor's Drive) provides a more initially attractive route as per our ISS heuristic: Commonwealth Ave. is long and straight and heads in the general direction (north) of the destination. On the other hand, Route 2 (Massachusetts Ave. to N. Pleasant St.) is less attractive, because the initial head-

Table 2

\begin{tabular}{|c|c|c|c|c|}
\hline $\begin{array}{c}\text { Percenta } \\
\text { Initial S } \\
\text { Chanc } \\
\text { on the } \\
\end{array}$ & $\begin{array}{l}\text { Rout } \\
\text { nt St } \\
\text { ondi } \\
\text { iffer }\end{array}$ & $\begin{array}{l}\text { Ces } C \\
\text { and } \\
\text { oss } \\
\text { mpu }\end{array}$ & $\begin{array}{l}\text { nt W } \\
\text { ted } P \\
\text { s for } \\
\text { in } E x\end{array}$ & $\begin{array}{l}\text { of the } \\
\text { ige of } \\
\text { oute } \\
\text { ent } 5\end{array}$ \\
\hline & & & & \\
\hline Map & $\mathrm{N} / \mathrm{E}$ & $\mathrm{S} / \mathrm{W}$ & Mean & Chance \\
\hline Clemson & 85 & 41 & 63 & 21 \\
\hline Hartford & 48 & 53 & 51 & 48 \\
\hline Alabama & 33 & 83 & 58 & 21 \\
\hline Adelphi & 60 & 33 & 47 & 32 \\
\hline Amherst & 56 & 66 & 61 & 48 \\
\hline
\end{tabular}

ing is south, which is in the opposite direction to the destination. These predictions were confirmed by our data; the subjects heading from A to B selected Route 1 over Route 2 by a two-to-one margin ( $66 \%$ vs. $34 \%)$. When heading from Point B to A, the attractiveness of the potential routes is reversed. Route 2 becomes the preferred route, because it is initially long and straight and heads south, in the general direction of the destination. Route 1 , on the other hand, heads west for a bit and then enters a turn where the subjects have to go north, in the opposite direction to the destination. In this case, the subjects selected Route 2 over Route 1 ( $56 \%$ vs. $44 \%$ ). Overall, the subjects selected our predicted ISS route $61 \%$ of the time for this particular map (chance responding $=48 \%$ ).

The previous example involved routes of similar length. The ISS effect is particularly striking when we examine maps on which there was a choice between a route that was clearly shorter versus an alternative that was more IS. In two cases (Clemson and Alabama), there was a single route that was overwhelmingly preferred by the subjects in either direction. However, the proportion of the subjects who chose the less optimal alternative varied significantly as a function of which direction the subject was traveling in, as would be predicted by the ISS heuristic.

As an example, consider the schematized version of the Clemson campus map that shows the two ISS routes (see Figure 7). Notice that, between Points A and B, there are a number of possible routes. The two we were primarily interested in were Fort Hill St.-Calhoun Drive (Route 1) and Williamson Rd.-Rte. 93 (Route 2). Route 1 is the more optimal alternative, since it is $20 \%$ shorter than Route 2 $(7.47 \mathrm{~cm}$ vs. $8.89 \mathrm{~cm})$. It is also the ISS route when traveling from Building A to Building B. On the other hand, Route 2, although longer, is initially more straight when traveling from B to A, since Calhoun Drive initially heads away from the destination. This was reflected in our results. Although, Route 1 was chosen almost $70 \%$ of the time overall, it was chosen $85 \%$ of the time when it was the ISS route from Point A to B and only $54 \%$ of the time when it was not the ISS route (from B to A). Likewise, Route 2 was chosen $41 \%$ of the time when it was the ISS route and only $12 \%$ of the time when it was not (chance responding $=21 \%$ ).

In summary, Experiment 5 demonstrated that subjects made asymmetric route choices when planning routes on campus maps. Furthermore, these asymmetries appear to stem from the use of the ISS, subjects tended to choose routes that were initially straight. Consequently, we were able to predict asymmetries, even when there was a strong preference for a single route. These results are especially compelling since the stimuli were actual maps. Thus, the previous results, which were obtained with artificial maps, do seem to generalize to the kinds of maps that people use in everyday route planning.

\section{GENERAL DISCUSSION}

The results demonstrate clearly that asymmetries in route selection occur reliably and across a variety of con- 


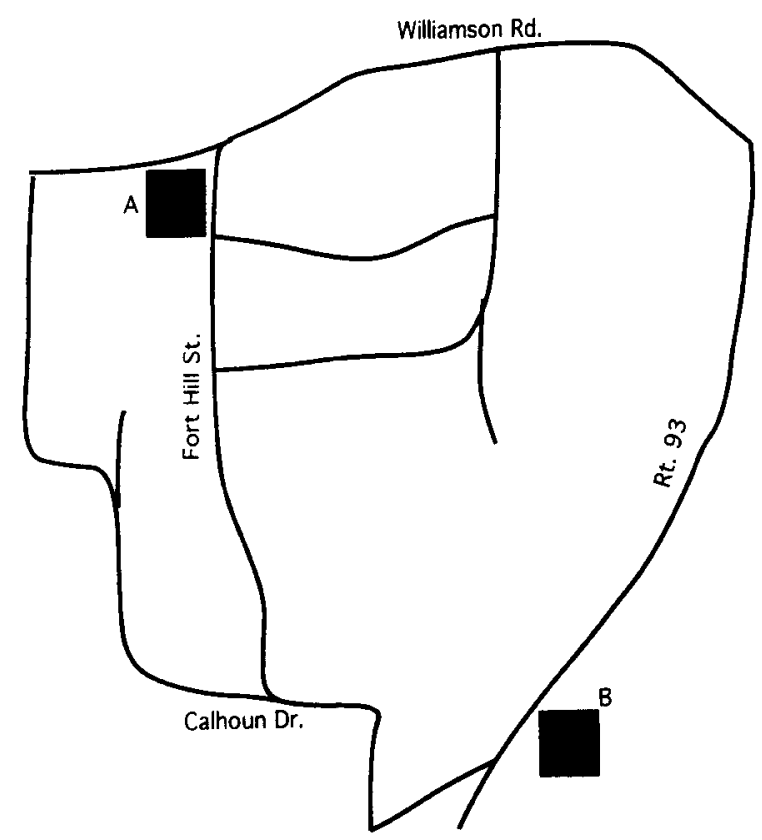

Figure 7. A schematized version of the Clemson map from Experiment 5. The top of the page is north.

texts. In the present research, we have identified asymmetries in route selection for different types of maps; previous research has demonstrated the effect in pedestrian navigation across a college campus (Shum et al., 1998). The results thus show that asymmetries in route selection occur both in the real world and in experimental contexts.

Our findings also suggest that the occurrence of asymmetries can be reliably predicted. The present results support the claim that asymmetries stem from a general heuristic regarding how people make route decisions. Specifically, people have clear preferences regarding the characteristics of initial route segments that influence their decisions, and the preferred initial route segment will often be different when traveling in one direction than when traveling in the opposite direction. Put simply, people's choices are asymmetric because they are overly influenced by characteristics of the initial portions of routes. The present results help to delineate the conditions under which people rely on this heuristic.

Experiment 1 demonstrated that subjects prefer to travel on a straight route more often than on a route with turns, even when the two routes are equal in length. Experiments 2 and 3 showed that this preference for straight routes is most pronounced in the initial segment of the route. In Experiment 2 , people attended to the straightness of a route more often when the straight portion was at the beginning of the route. The subjects chose different routes when they traveled in opposite directions between Point $\mathrm{A}$ and Point $B$ when the initial segments of the routes were differentially straight. In Experiment 3, we manipulated the subjects' attention to the initial portion of routes by imposing region boundaries on the maps. We were able to shift peo- ples' route choices by placing region boundaries so that routes either included or excluded turns in the initial region, although the actual characteristics of the possible routes themselves remained unchanged.

The results of Experiments 4 and 5 help to refine our explanation of when and why people rely on the ISS. The subjects used the ISS more often when they were under high cognitive load (Experiment 4) and when choosing routes on real, geographic maps of college campuses (Experiment 5). In Experiment 4, we increased the subjects' mental load by imposing time constraints while they examined the maps. The subjects relied more on the ISS when mental load was high than when it was relatively low. However, we showed that subjects could disregard the heuristic and choose the optimal route (even under a time constraint) when they were motivated to do so. In Experiment 5, we extended the findings of the other studies by demonstrating that the subjects used the ISS when choosing routes on real maps of college campuses.

Our results are similar to those of other studies that have found that the use of heuristics in problem solving can lead to asymmetries or systematic biases. Although heuristics such as the ISS can lead to inaccuracies or asymmetries, they can also help to reduce the amount of information that must be processed to make a decision (Hogarth, 1981; Kleinmuntz \& Kleinmuntz, 1981). The ISS leads to predictable asymmetries that might be considered nonoptimal in terms of minimizing travel distance or time. However, in many cases, one would expect the heuristic to lead to reasonably quick routes with significantly less cognitive effort than would be required to do an exhaustive search for the optimal route.

Our results, therefore, are consistent with the more general notion that asymmetries or distortions in spatial judgments do not necessarily reflect limitations in the processing or representation of spatial information. We found that people strongly prefer to select a route that is IS, even if there is a shorter alternative. At the same time, however, we demonstrated that people could reliably choose the shortest route when they were instructed to do so (Experiment 4). Perhaps, in many situations, the time that would be needed to plan the route may negate the advantage that would be gained in travel time. The ISS may in fact be the best compromise between time needed to plan an optimal route and the extra time required to travel a less-than-optimal route. Other researchers have reached similar conclusions regarding, for example, distortions and asymmetries in distance judgments (McNamara \& Diwadkar, 1997; Newcombe et al., 1996).

Our results raise the question of whether reliance on the ISS stems from perceptual factors. Does a route with an initially straight segment actually appear shorter than other routes? We believe, for two reasons, that the ISS represents a preference for the initially straighter route and not a perceptual bias to see straight routes as shorter than circuitous routes. First, as was mentioned above, we demonstrated in Experiment 4 that people could put aside the ISS and choose the shortest route, even under cognitive load, when 
they were instructed to do so. That people are able to disregard the ISS when motivated suggests that the initially straight route does not necessarily appear to be shorter.

Second, we have conducted additional pilot studies to assess subjects' perceptual judgments of the lengths of the routes. In this research, we asked subjects to rate the lengths of the IS, IC, and SHO routes that we used in Experiment 4 . Subjects consistently rated the SHO route as shorter than the other two, but most important, they rated the IC and IS routes as similar in length, even when the two were presented briefly $(750 \mathrm{msec})$. Taken together, these results support the claim that perceptual factors alone are unlikely to explain people's reliance on the ISS.

We suggest instead that the ISS may reflect the process by which people go about planning routes in real-world contexts. In an attempt to reduce cognitive effort, subjects may actually infer that a route beginning with a straight segment is shorter than a route that does not. According to this explanation, people may compare only the initial segments of competing routes and generalize the characteristics of those initial segments to the rest of the routes. In this sense, the effect is more psychological than perceptual; people do not actually perceive initially straight routes as being shorter but may think that they should be.

In addition, the process of route choice may be incremental, in that rather than plan the shortest or most optimal route between two locations, people may, instead, break down the task of traveling between two locations into a series of step-by-step decisions. They may follow a particular segment until a decision must be made, usually at a turn or a juncture (Christenfeld, 1995). This possibility is consistent with previous work (Bailenson et al., 1998; Downs et al., 1988; Sadalla et al., 1980) that has shown that, when traveling in cluttered, complex areas, people tend to plan routes on a region-by-region basis. Most of the time, the route within each region with the straightest initial segment at each juncture will also be the shortest segment as well, although, as we have demonstrated here, this is not always true.

In sum, the present work offers a new explanation for a seemingly counterintuitive finding: People often select different routes, depending on the direction of travel. We were able to account for these asymmetries in terms of the general cognitive principle that people rely on heuristics to minimize cognitive effort. Route planning, like many other cognitive tasks, can be difficult and time consuming. Heuristics such as the ISS give us a way to make reasonable guesses without expending the effort needed to find an optimal solution.

\section{REFERENCES}

Ballenson, J. N., Shum, M. S., \& UtTal, D. H. (1998). Road climbing: Principles governing asymmetric route choices on maps. Journal of Environmental Psychology, 18, 251-264.
BOVy, P. H. L., \& STERn, E. (1990), Route choice: Wayfinding in transport networks. Dordrecht, Kluwer.

Christenfeld, N. (1995). Choices from identical options. Psychological Science, 6, 50-55.

DENIS, M., \& CoCUdE, M. (1989). Scanning visual images generated from verbal descriptions. European Journal of Cognitive Psychology, 1, 293-307.

Downs, R. M., Lisen, L. S., \& DagGs, D. G. (1988). On education and geographers: The role of cognitive development theory in geographic education. Annals of the Association of American Geographers, 78 , 680-700.

DunNing, D., \& PARPal, M. (1989). Mental addition versus subtraction in counterfactual reasoning: On assessing the impact of personal actions and life events. Journal of Personality \& Social Psychology, 57, 5-15.

Hogarth, R. M. (1981). Beyond discrete biases: Functional and dysfunctional aspects of judgmental heuristics. Psychological Bulletin, 90, 197-217.

HoLYOAK, K. J., \& MAH, W. A. (1982). Cognitive reference points in judgments of symbolic magnitude. Cognitive Psychology, 14, 328-352.

KleinmuntZ, D. N., \& KleinmunTZ, B. (1981). Decision strategies in simulated environments. Behavioral Science, 26, 294-305.

McNamaRa, T. P. (1991). Memory's view of space. In G. H. Bower (Ed.), The psychology of learning and motivation: Advances in research and theory (Vol. 27, pp. 147-186). San Diego: Academic Press.

McNamara, T. P., \& Diwadkar, V. A. (1997). Symmetry and asymmetry of human spatial memory. Cognitive Psychology, 34, 160-190.

MONTELLO, D. R. (1991). The measurement of cognitive distance: Methods and construct validity. Journal of Environmental Psychology, 11 , 101-122.

Newcombe, N., Huttenlocher, J., Sandberg, E., \& Lie, E. (1996, October). What do asymmetries in judgment indicate about representation? The case of spatial estimation. Presented at the 37th Annual Meeting of the Psychonomic Society, Chicago.

Payne, J. W., Bettman, J. R., \& Johnson, E. J. (1988). Adaptive strategy selection in decision making. Journal of Experimental Psychology: Learning, Memory, \& Cognition, 14, 534-552.

RoBIN, F., \& DENIS, M. (1991). Description of perceived or imagined spatial networks. In R. H. Logie \& M. Denis (Eds.), Mental images in human cognition (pp. 141-152). Amsterdam: Elsevier

Sadalla, E. K., Burroughs, W. J., \& Staplin, L. J. (1980). Reference points in spatial cognition. Journal of Experimental Psychology, 6 516-528

Seneviante, P. N., \& Morrall, J. F. (1986). Analysis of factors affecting the choice of route of pedestrians. Transportation Planning \& Technology, 10, 147-159.

Shum, M. S., Bailenson, J. N., Hwang, S., Piland, L., \& Uttal, D. (1998). Road climbing: A route choice heuristic. In Proceedings of the 20th Annual Conference of the Cognitive Science Society (pp. 667. 673). Mahwah, MJ: Erlbaum.

Simon, H. A. (1981). The sciences of the artificial. Cambridge, MA: MIT Press.

STERN, E., \& LEISER, D. (1988). Levels of spatial knowledge and urban travel modeling. Geographical Analysis, 20, 140-155.

TVersky, A. (1977). Features of similarity. Psychological Review, 84, $327-352$.

\section{NOTES}

1. On all five experiments discussed in this paper, there were no ef fects of gender or handedness. Consequently, we will not discuss these factors.

(Manuscript received July 2, 1998 revision accepted for publication March 3, 1999.) 